\title{
Entropy-information perspective to radiogenic heat distribution in continental crust
}

\author{
R. N. Singh and A. Manglik \\ CSIR-National Geophysical Research Institute, Uppal Road, Hyderabad 500007, India
}

Correspondence to: R. N. Singh (rishiprema@gmail.com)

Received: 11 March 2013 - Revised: 14 May 2013 - Accepted: 27 May 2013 - Published: 26 June 2013

\begin{abstract}
Depth distribution of radiogenic heat sources in continental crust is an important parameter that controls its thermal structure as well as the mantle heat flow at the base of continental lithosphere. Various models for the depth distribution of radiogenic heat sources have been proposed. Starting from constant and exponential models based on linear heat flow-heat generation relationship the present-day layered models integrate crustal structure and laboratory measurements of radiogenic heat sources in various exposed rocks representing crustal composition. In the present work, an extended entropy theory formalism is used for estimation of radiogenic heat sources distribution in continental crust based on principle of maximum entropy (POME). The extended entropy principle yields a constant heat generation model if only a constraint given by total radiogenic heat in the crust is used and an exponential form of radiogenic heat sources distribution if an additional constraint in the form of a second moment is used in the minimization of entropy.
\end{abstract}

\section{Introduction}

Radiogenic heat sources in continental crust contribute significantly to the observed surface heat flow and thermal structure of the continental lithosphere, which is linked to a variety of tectonic and geochemical processes. Their depth distribution, however, is a major source of uncertainty in thermal models of the continental lithosphere and estimates of the mantle heat flow beneath continents. The studies in the late sixties and early seventies focused on devising methods to estimate the depth distribution of these sources based on measurements of the concentration of radiogenic heat sources in exposed rocks (or shallow borehole samples) and correlating these with the estimates of surface heat flow values for a geological province. The discovery of a linear relationship between surface heat flow $(Q)$ and heat generation $(A)$ by Birch et al. (1968), Roy et al. (1968) and Lachenbruch (1968, 1970) provided a well-constrained approach for the estimation of the Moho heat flow and an idea about the depth distribution of radiogenic sources. In this relationship, the intercept of the line (heat flow when $A$ is zero) gives reduced heat flow and slope provides information about the depth extent of distribution of radiogenic sources.

Using this linear heat flow-heat generation $(Q-A)$ relationship, Birch et al. (1968) proposed a step model for the depth distribution of radiogenic sources in which the thickness of the step is equal to the slope of the linear fit. Lachenbruch (1970) demonstrated that an exponential model with logarithmic decrement equal to the slope also satisfies the $Q-$ $A$ relationship. He argued that such an exponential distribution would yield linear relationship in the presence of differential erosion of the continental crust. Singh and Negi (1979) and Lachenbruch (1980) showed that many more interpretations of the $Q-A$ relationship are possible depending on the nature of the Moho heat flow and crustal thickness.

The exponential model of depth distribution of radiogenic sources has also been justified from several physical considerations. Turcotte and Oxburgh (1972) presented a statistical thermodynamic model showing that radioactive species would move upward, along with other species in the gravitational field, yielding an exponential model of radiogenic sources. Albarede (1975) presented a dissolution-precipitation model of the crystallizing and cooling magmatic bodies yielding an exponential model. Buntebarth (1976) showed that uranium concentration in granitic bodies is controlled by the processes of advection and diffusion and suggested that both these processes applied to crust would be able to produce an exponential distribution of 
radiogenic sources. Singh and Negi (1980a, b) constructed a model of depth distribution of radiogenic sources by minimizing the rate of entropy production and smoothness of the radiogenic heat profiles to show that it leads to an exponential model. This model was extended to include the advection of heat by fluid flows by Cermak and Bodri (1993), Bodri and Cermak (1993) and Singh and Manglik (2000). Singh (1981) has used the Pontryagin principle to show that an exponential model is an optimal model.

Next stage in the proving models of the depth distribution of radiogenic sources was taken when petrological models of the crust became definitive. Seismic images of the compression terrains show that the rocks at depth are exposed at the surface. Thus, from surface exposures of the rocks, depth distribution of rock compositions have been worked out for several continents (Nicolaysen et al., 1981; Roy et al., 2008). Concentration of radioactive elements in exposed rocks formed under different pressure-temperature conditions provided models for depth distribution of radioactive elements under the assumption that no further differentiation of radioactive elements took place since the major crust forming events. Such a reconstruction of depth distribution of radiogenic heat sources yields a discontinuous step model rather than an exponential model. These models produced a thermal structure of the continental crust much cooler than that obtained by exponential model (Drury, 1985; Ashwal et al., 1987).

Another approach to estimating the depth distribution of radiogenic sources, considered as non-random, is through the application of extended entropy information theoretic models (Kapur and Kesavan, 1992; Woodbury, 2012). In an interesting study, Singh (2010) used entropy maximization methods to constrain various infiltration models. Woodbury (2012) has clarified some subtle issues about the number of constraints used in the Singh's formulation in deriving the infiltration equation. In this paper we will use Woodbury's formalism to constrain the depth distribution of radiogenic sources.

\section{Principle of maximum entropy (POME)}

This principle was enunciated to obtain probability distribution function (pdf) for a random variable under prescribed constraints. The Shannon entropy of the pdf was maximized under given constraints in order to obtain the least biased pdf of a random variable (Jaynes, 1957). We follow this approach to obtain the depth distribution of radiogenic sources in the crust by maximizing entropy of the heat production function (given a few constraints on the heat production) using the standard Lagrangian multiplier method. We follow the recent work of Woodbury (2012) in deriving the form of radiogenic heat in the crust. We first take the case of one constraint and then extend the concept to two constraints. It is interesting that the two popular models, step and exponential models, of the radiogenic sources for the crust follow from this formulation.

\section{Mathematical formulation and solution}

The one-dimensional steady state heat conduction equation including the radiogenic heat sources is given as

$K \frac{d^{2} T^{\prime}}{\mathrm{d} z^{\prime 2}}+A^{\prime}\left(z^{\prime}\right)=0$

where $T^{\prime}$ is the temperature, $K$ is the thermal conductivity assumed to be constant, $A^{\prime}$ is the radiogenic heat sources, and $z^{\prime}$ is the depth coordinate positive downward. In general, prescribed surface heat flux and temperature are taken as boundary conditions to solve Eq. (1) for temperature distribution within the crust for known distribution of radiogenic heat sources. We use these boundary conditions and an additional boundary condition of prescribed mantle heat flux. These boundary conditions are

$T^{\prime}\left(z^{\prime}=0\right)=T_{\mathrm{S}}, \quad q\left(z^{\prime}=0\right)=q_{\mathrm{S}}$,

$q\left(z^{\prime}=L\right)=q_{L}$,

where $q_{\mathrm{s}}$ and $q_{L}$ are surface and mantle heat flux and $T_{\mathrm{S}}$ is the surface temperature. The values of surface heat flow as well as surface heat generation are observed. Mantle heat flow can be estimated from the Birch-Lachenbruch $Q-A$ relationship, as discussed above. Empirical studies have shown that mantle contributes to about $60 \%$ of the total surface heat flow (Pollack and Chapman, 1977). Thus, some estimates of the mantle heat flow can be obtained. Mantle heat flow can also be estimated from mantle convection models. Theoretical calculations on heat transport in convecting mantle yield values of the Moho heat flow (Korenaga, 2003, 2008). We can use these results constraining the mantle heat flow.

The above equations are non-dimensionalized by using $z=z^{\prime} / L, A=A^{\prime} / A_{\mathrm{S}}, T=\left(T^{\prime}-T_{\mathrm{S}}\right) /\left(T_{L}-T_{\mathrm{S}}\right)$ and $q_{0}=$ $K\left(T_{L}-T_{\mathrm{S}}\right) / L$, where $L$ is the depth at which temperature is $T_{L}$, which can be considered as the depth to the lithosphereasthenosphere boundary or the depth to the Moho. Here, we assume that the sub-crustal lithosphere does not contain any radiogenic heat sources and therefore consider $L$ to be the depth to the Moho and prescribe mantle heat flux $q_{L}$ at this depth. Although $T_{L}$ is used to non-dimensionalize the heat conduction equation, it is an unknown parameter that can be determined once the solution for $T^{\prime}\left(z^{\prime}\right)$ is obtained.

In non-dimensional form we get

$\frac{d^{2} T}{\mathrm{~d} z^{2}}+\alpha A(z)=0$,

with boundary conditions

$\left.\frac{\mathrm{d} T}{\mathrm{~d} z}\right|_{z=0}=q_{\mathrm{S}} / q_{0}=q_{a},\left.\quad \frac{\mathrm{d} T}{\mathrm{~d} z}\right|_{\mathrm{z}=1}=q_{L} / q_{0}=q_{b}$, 
$T(z=0)=0$,

$T(z=1)=1$,

where $\alpha=\mathrm{LA} / q_{0}$.

Integrating Eq. (3) over the thickness of the layer and applying boundary conditions Eq. (4a), we get

$\int_{0}^{1} A(z) \mathrm{d} z=\frac{1}{\alpha}\left(q_{a}-q_{b}\right)=p_{0}$.

Further, integrating Eq. (3) and applying boundary conditions Eq. (4b), we get:

$\int_{0}^{1} z A(z) \mathrm{d} z=\frac{1}{\alpha}\left(1-q_{b}\right)=p_{1}$.

Equations (5) and (6) are constraints on $A(z)$. Equation (5) gives total heat generation in the crust and Eq. (6) depends on the prescribed value of mantle temperature.

\subsection{Case 1: one constraint known}

We write the following expression for the entropy of $A(z)$ as

$S=-\int_{0}^{1} A(z) \ln (A(z)) \mathrm{d} z$

In applying the principle of maximum entropy, we maximize $S$ given constraints on $A(z)$, such as those given in Eqs. (5) and (7). The expression for $A(z)$ is obtained using the method of Lagrangian multipliers. We thus need to maximize the following function with respect to $A(z)$ :

$H=-\int_{0}^{1} A(z) \ln (A(z)) \mathrm{d} z-\lambda_{0}\left[\int_{0}^{1} A(z) \mathrm{d} z-p_{0}\right]$.

In this case, we assume that only the surface and mantle heat fluxes are known. Maximizing $H$, we get after taking the fist derivative of $H$ with respect to $A$ as

$\frac{\partial H}{\partial A}=-[1+\ln (A(z))]-\lambda_{0}=0$.

We thus get

$A(z)=\exp \left[-\left(1+\lambda_{0}\right)\right]$.

Since the above expression does not depend on $z$, this represents a constant radiogenic heat source distribution model of the form $A(z)=A_{0}$. Further, as $A_{0}=1$ in the nondimensional form, we get $\lambda_{0}=-1$. This implies that with only prescribed heat flux condition (Eq. 4a), the entropy approach supports the constant heat generation model. In this case the heat generation is zero from the base of step model to the Moho and $q_{L}$ is the heat flux at the base of the radiogenic layer.

\subsection{Case 2: two constraints known}

Here, we assume that both heat flux and temperature are known at the two boundaries and define maximization criteria as

$$
\begin{aligned}
H & =-\int_{0}^{1} A(z) \ln (A(z)) \mathrm{d} z-\lambda_{0} \\
& {\left[\int_{0}^{1} A(z) \mathrm{d} z-p_{0}\right]-\lambda_{1}\left[\int_{0}^{1} z A(z) \mathrm{d} z-p_{1}\right] . }
\end{aligned}
$$

On maximizing $H$ with respect to $A$ we get

$\frac{\partial H}{\partial A}=-[1+\ln (A(z))]-\lambda_{0}-\lambda_{1} z=0$.

This gives $A(z)=\exp \left[-\left(1+\lambda_{0}+\lambda_{1} z\right)\right]$ which is of the form $A(z)=A_{0} \exp [-z / d]$ where $A_{0}=\exp \left[-\left(1+\lambda_{0}\right)\right]$ and $d=1 / \lambda_{1}$. As $\lambda_{0}=-1$, the final expression for the radiogenic heat sources distribution reduces to

$A(z)=\exp \left[-\lambda_{1} z\right]$,

substituting Eq. (13) into Eqs. (5) and (6) we get two equations

$$
\begin{array}{r}
\exp \left(-\lambda_{1}\right)+p_{0} \lambda_{1}-1=0, \\
\left(1+\lambda_{1}\right) \exp \left(-\lambda_{1}\right)+p_{1} \lambda_{1}^{2}-1=0 .
\end{array}
$$

The above two equations have one unknown $\lambda_{1}$, if we assume that the temperature $T_{L}$ at the lower boundary is known. In case of $T_{L}$ unknown both $p_{0}$ and $p_{1}$ are unknown variables and we now have three unknown parameters. However, substituting the values of $\alpha$ and $q_{a}, q_{b}$ into Eq. (5) gives $p_{0}=\left(q_{\mathrm{S}}-q_{L}\right) / \mathrm{LA}$ S which is not a function of $T_{L}$. Therefore, Eq. (14a) can be solved numerically to find its root for different values of $p_{0}$. Figure 1 shows variation of $\lambda_{1}$ with $p_{0}$. Here, dashed line shows the value of $\lambda_{1}$ for which heat sources at the base of the model (Moho) become 10 percent of the surface value.

Once the value of $\lambda_{1}$ is obtained, the expression for $p_{1}$ in Eq. (6) can be substituted into Eq. (14b) to get an expressions for the temperature at $(z=L)$ which is

$T_{L}=T_{\mathrm{S}}+\frac{L}{K}\left[\left(\frac{1+\lambda_{1}}{\lambda_{1}}\right) q_{\mathrm{S}}-\frac{1}{\lambda_{1}}\left(q_{L}+\mathrm{LA}_{\mathrm{S}}\right)\right]$,

to calculate the temperature $T_{L}$.

The system of Eqs. (3), (4a) and (4b) can be solved to get an analytical expression for temperature in non-dimensional form as a function of depth as

$T(z)=\left(q_{a}-\frac{\alpha}{\lambda_{1}}\right) z+\frac{\alpha}{\lambda^{2}}\left(1-\exp \left(-\lambda_{1} z\right)\right)$. 


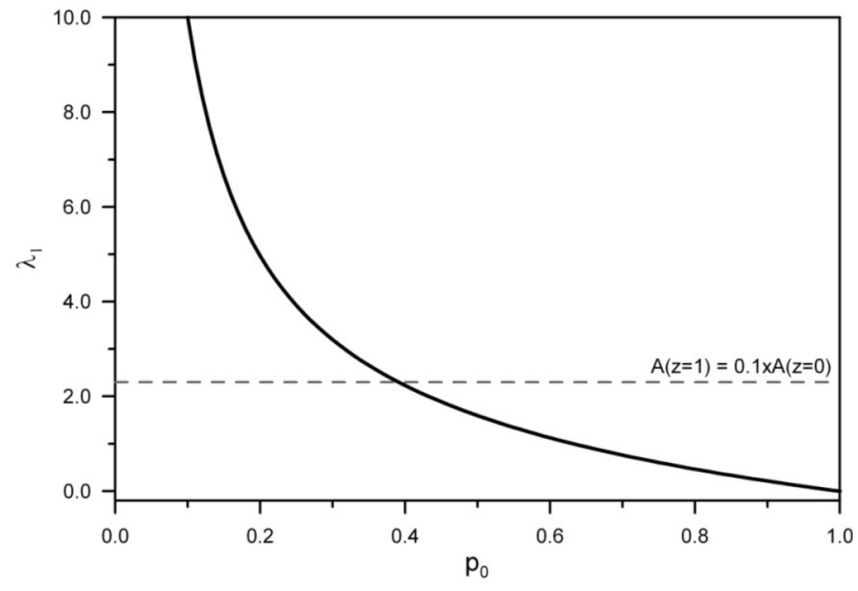

Fig. 1. Variation of $\lambda_{1}$ with $p_{0}$. Dashed line represents the values of $\lambda_{1}$ and $p_{0}$ for which radiogenic heat source at the Moho decreases to 10 percent of its surface value.

Converting Eq. (16) to dimensional form and defining $d=$ $L / \lambda_{1}$, the expression for temperature becomes

$T(z)=T_{\mathrm{S}}+\frac{1}{K}\left[\left(q_{\mathrm{S}}-A_{\mathrm{S}} d\right) z+A_{\mathrm{S}} d^{2}(1-\exp (-z / d))\right]$.

The entropy principle thus supports an exponential form of radiogenic heat sources distribution if first and second moments are used in the minimization of entropy.

\section{Discussion}

As an example, we take crustal thickness $40 \mathrm{~km}$, thermal conductivity $3 \mathrm{~W}(\mathrm{~m} \mathrm{~K})^{-1}$, surface heat flux $40 \mathrm{~mW} \mathrm{~m}^{-2}$, surface heat generation $1.3 \mu \mathrm{W} \mathrm{m}{ }^{-3}$, and mantle heat flux as 0.6 times the surface heat flux (Pollack and Chapman, 1977). For these values $p_{0}=0.3$ and $\lambda_{1}=3.1971$. This corresponds to the exponential decay constant $d=12.5 \mathrm{~km}$ and gives Moho temperature of $377^{\circ} \mathrm{C}$. The concentration of radiogenic heat sources at the Moho is $4 \%$ of its surface value. In another example, we take the heat flow-heat generation data of Basin and Range Province. Blackwell (1971) used a two-layered crustal model of this region having surface heat flow of $79 \mathrm{~mW} \mathrm{~m}^{-2}$ with $10 \mathrm{~km}$-thick upper crust having radiogenic heat sources concentration of $2.1 \mu \mathrm{W} \mathrm{m}{ }^{-3}$. The lower crust of $20 \mathrm{~km}$ thickness was assumed to be free of radiogenic heat sources. This yields mantle heat flow of $57 \mathrm{~mW} \mathrm{~m}^{-2}$ assuming mantle heat generation to be negligible. For these values of parameters we get $p_{0}=0.333$ and $\lambda_{1}=2.8215$ and corresponding exponential decay constant $d=10.6 \mathrm{~km}$.

The above formulation is valid for a single-layer crust in which the radiogenic heat source concentration exponentially decreases with depth. For a geological province, e.g., cratonic region, the contribution of mantle heat flux to the surface heat flux values remains spatially fairly uniform. Any short-wavelength variations in the mantle heat flux get
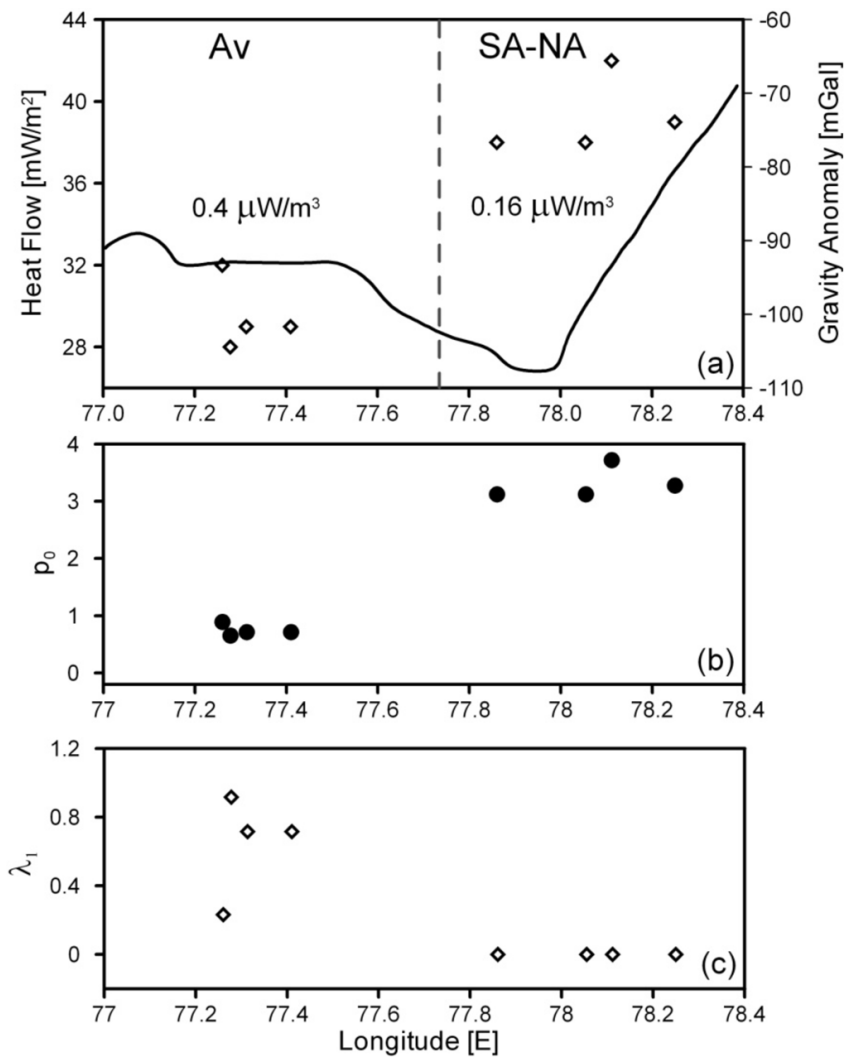

Fig. 2. (a) Surface heat flow and heat generation values along with Bouguer gravity anomaly for PCSZ (from Manglik, 2006), and corresponding values of (b) $p_{0}$ and (c) $\lambda_{1}$.

attenuated over the thickness of the lithosphere. Therefore, the above formulation, under 1-D assumption, can be used to estimate the spatial variations in the radiogenic heat contribution of the crust to the surface heat flow for a geological province.

In some geological situations, we may have to invoke multi-layer models each having a different level of heat sources concentration to satisfy the surface and Moho heat flow. This is demonstrated by an example using heat flowheat generation (HF-HG) data from Palghat Cauvery Shear Zone (PCSZ), India. Manglik (2006) analyzed HF-HG data from the northern part of PCSZ in conjunction with the crustal structure obtained by integrated geophysical studies. In this region, the surface heat flow falls under two clusters separated by an average distance of less than $100 \mathrm{~km}$ (Fig. 2a) and average surface heat generation is also distinct. $\mathrm{He}$ obtained the mantle heat flow value of $17 \mathrm{~mW} \mathrm{~m}^{-2}$ for this region and attributed the spatial variations in the surface heat flow to the variations in the contribution of crustal heat generation. We calculate $p_{0}$ and $\lambda_{1}$ for this data set. The results are shown in Fig. $2 b$ and c. The value of $\lambda_{1}$ is about 0.8 for the cluster Avinashi (Av) whereas it is almost zero for the Salem-Namakkal (SA-NA) cluster (Fig. 2c) indicating that the radiogenic heat sources are almost constant within the 
entire layer (see Eq. 13). These values violate the assumption that there are no radiogenic heat sources in the subcrustal mantle (depth larger than $L$ ) because of slow (Av) or almost no (SA-NA) decrease in the concentration of radiogenic sources with depth. The value of radiogenic heat source at the depth $L$ should decrease appreciably from it surface value for the solution to remain valid. The dashed line in Fig. 1 presents one such limit for which $\lambda_{1}$ is 2.30258. Since the values of $\lambda_{1}$ for Av and SA-NA are much smaller than this value, we infer the presence of some other layer within the crust having much larger concentration of the radiogenic heat sources in order to balance the net heat transfer across the crust. This corroborates with Manglik (2006) result in which he attributed high concentration of radiogenic sources to the low-velocity mid-crustal layer, assuming it to be having upper crustal affinity under the framework of the continental collision model proposed for PCSZ.

\section{Conclusions}

The principal of extended maximum entropy applied to the steady state heat conduction equation for the estimation of radiogenic heat sources distribution in continental crust yields constant heat generation model only if first moment is used and an exponential model if both first and second moments are used in the minimization of entropy. The thermal models that rely on the known values of the surface temperature, surface heat flow, and surface radiogenic heat generation thus lead to an exponential model for radiogenic sources satisfying entropy constraint.

Acknowledgements. The work has been carried out under an INSA Senior Fellowship to RNS. Contribution under CSIR project PSC0204.

Edited by: L. Telesca

Reviewed by: two anonymous referees

\section{References}

Albarede, F.: The heat flow/heat generation relationship: An interaction model of fluids with cooling intrusions, Earth Planet. Sci. Lett., 27, 73-78, 1975.

Ashwal, L. D., Morgan, P., Kelly, S. A., and Preicival, G. A.: Heat production in an Archean crustal profile and implications for heat flow and mobilization of heat producing elements, Earth Planet. Sci. Lett., 85, 439-450, 1987.

Birch, F., Roy, R. F., and Decker, E. R.: Heat flow and thermal history in New York and New England, in: Studies of Appalachian Geology: Northern and Maritime, edited by: Zen, E-An., White, W. S., Hadley, J. B., and Thompson Jr., J. B., Interscience, New York, 437-451, 1968.

Blackwell, D. D.: The thermal structure of the continental crust, in: The structure and physical properties of the earth's crust, edited by: Heacock, J. G., Geophys. Monograph 14, 169-184, 1971.
Bodri, L. and Cermák, V.: Heat production in the continental lithosphere, part II: variational approach, Tectonophysics, 225, 2934, 1993.

Buntebarth, G.: Distribution of uranium in intrusive bodies due to combined migration and diffusion, Earth Planet. Sci. Lett., 32, 84-90, 1976.

Cermak, V. and Bodri, L.: Heat production in the continental crust, part I: data converted from seismic velocities and their attempted interpretation, Tectonophysics, 225, 15-28, 1993.

Drury, M. J.: Heat flow and heat generation in the Churchill Province of the Canadian Shield, and their paleotectonic significance, Tectonophysics, 115, 25-44, 1985.

Jaynes, E. T.: Information theory and statistical mechanics, Phys. Rev., 106, 620-630, 1957.

Kapur, J. N. and Kesavan, H. K.: Entropy optimization principles with applications, Academic Press, 408 pp., 1992.

Korenaga, J.: Energetics of mantle convection and the fate of fossil heat, Geophys. Res. Lett., 30, 1437, doi:10.1029/2003GL016982, 2003.

Korenaga, J.: Urey ratio and the structure and evolution of Earth's mantle, Rev. Geophys., 46, 1-32, doi:10.1029/2007RG000241, 2008.

Lachenbruch, A. H.: Preliminary Geothermal Model of the Sierra Nevada, J. Geophys. Res., 73, 6977-6989, doi:10.1029/JB073i022p06977, 1968.

Lachenbruch, A. H.: Crustal temperature and heat production: Implication of the linear heat flow relation, J. Geophys. Res., 75, 3291-3300, 1970.

Lachenbruch, A. H.: Comment on "A reinterpretation of the linear heat flow and heat production relationship for the exponential model of the heat production in the crust" by R. N. Singh and J. G. Negi, Geophy. J. Roy. Astron. Soc., 63, 791-795, 1980.

Manglik, A.: Mantle heat flow and thermal structure of the northern block of Southern Granulite Terrain, India, J. Geodynamics, 41, 510-519, 2006.

Nicolaysen, L. O., Hart, R. J., and Gale, N. H.: The Vredefort radioelement profile extended to supracrustal strata at Carletonville, with implications for continental heat flow, J. Geophys. Res., 86, 10653-10661, 1981.

Pollack, H. and Chapman, D.: On the regional variation of heat flow, geotherms, and lithospheric thickness, Tectonophysics, 38, 279296, 1977.

Roy, R. F., Blackwell, D. D., and Birch, F.: Heat generation of plutonic rocks and continental heat flow provinces, Earth. Planet. Sci. Lett., 5, 1-12, 1968.

Roy, S., Ray, L., Bhattacharya, A., and Sirnivasan, R.: Heat flow and crustal thermal structure in the Late Archaean Clospet granite batholith, south India, Int. J. Earth Sci., 97, 245-256, 2008.

Singh, R. N.: On the application of the pontryagin's maximum principle for identification of the radiogenic heat distribution in the Earth's interior, Int. J. Eng. Sci., 19, 1601-1604, 1981.

Singh, R. N. and Manglik, A.: Identification of radiogenic heat source distribution in the crust: A variational approach, Sadhana, 25, 111-118, 2000.

Singh, R. N. and Negi, J. G.: A reinterpretation of the linear heat flow and heat production relationship for the exponential model of the heat production in the crust, Geophys. J. Roy. Astron. Soc., 57, 741-744, 1979. 
Singh, R. N. and Negi, J. G.: A variational approach to model the depth dependence of radiogenic heat in the crust, Geophys. Res. Lett., 7, 209-210, 1980a.

Singh, R. N. and Negi, J. G.: A variational approach to model the depth dependence of radiogenic heat in the crust - Addendum, Geophys. Res. Lett., 7, p. 559, 1980 b.

Singh, V. P.: Entropy theory for derivation of infiltration equations, Water Resour. Res., 46, W03527, doi:10.1029/2009WR008193, 2010.
Turcotte, D. L. and Oxbourgh, E. R.: Statistical thermodynamic model for the distribution of crustal heat sources, Science, 176, 1021-1022, 1972.

Woodbury, A. D.: Comment on "Entropy theory for derivation of infiltration equations" by Vijay P. Singh, Water Resour. Res., 48, W08801, doi:10.1029/2012WR012157, 2012. 